\title{
Mueller-matrix reconstruction of parameters of phase and amplitude anisotropy in diagnostics of endometriosis and infertility
}

\author{
A.G. Ushenko ${ }^{1}$, M.M. Dominikov ${ }^{1}$, I.I. Lakusta ${ }^{1}$, G.D. Koval ${ }^{2}$ \\ ${ }^{1}$ Chernivtsi National University, Optics and Publishing Department \\ 2, Kotsyubinsky str., 58012 Chernivtsi, Ukraine \\ ${ }^{2}$ Bukovinian State Medical University, 58000 Chernivtsi, Ukraine \\ E-mail: a.dubolazov@chnu.edu.ua
}

\begin{abstract}
A new azimuthally stable polarimetric method for processing microscopic images of optically anisotropic structures for different biological layers of histological sections has been proposed. A new model that enables to determine phase anisotropy of biological tissues by using superposition of Mueller matrices of linear birefringence and optical activity has been proposed. The matrix element $M_{44}$ has been chosen as the main information parameter, which value is independent of rotation angle of both sample and probing beam polarization plane.
\end{abstract}

Keywords: polarimetry, endometrium, laser image, filtering.

Manuscript received 05.11.14; revised version received 24.02.15; accepted for publication 27.05.15; published online 08.06.15.

\section{Introduction}

Development of computational methodologies for processing microscopic images is a new health-physical method. It is laser polarimetry of histological sections taken from biological tissues [1-3]. This method based on measurements of the coordinate distributions (polarization maps) in the plane of polarization states of histological sections microscopic images of biological tissues. This method provides a new, inaccessible to the histological and mathematical methods of analysis, information on the optical anisotropy (linear and circular birefringence) of multiscale structural elements of different biological objects.

At the same time, a complex analysis of the polarization maps of a tissue specimen is azimuthally dependent on the probing beam polarization plane and sample rotation angle. It makes it difficult to use this method in comparative research groups of histological sections with different pathologies. Thus, further progress of laser polarimetry may be related with the development of azimuthally stable methods of direct measuring the parameters of linear and circular birefringence.

Solution of this task lies in using Mueller-matrix mapping with the so-called rotational invariants. It has been shown in the works [4-6] that the matrix elements $M_{44}(\Theta)=$ const are azimuthally stable and independent of the sample rotation angle $(\Theta)$.

It has been determined that in the case of optically thin (extinction coefficient $\tau \leq 0.1$ ) layers, the $M_{44}(\Theta)$ value is preferably defined by phase anisotropy mechanisms - linear and circular birefringences [7-18].

Separation of these mechanisms $\left(M_{44} \Rightarrow\left(\begin{array}{c}M_{44}-\text { linear } \\ M_{44}-\text { circular }\end{array}\right)\right)$ is possible by using spatialfrequency Fourier selection [12, 13].

Our research is aimed at designing the experimental method of azimuthally stable Fourier 
polarimetry and spatial-frequency selection of parameter distributions for linear and circular birefringence in the healthy donor films and samples taken from the patients with endometriosis.

\section{Theory of the method}

The following model concepts have been assumed [1-3] by us as the basis for analyzing the processes of modulation of laser radiation by the network of biological layers with linear and circular birefringence:

- $\quad$ optical anisotropy of these structures is characterized by the distribution of the coordinate matrix element, where the direction of the optical axis is the phase shift between linearly polarized orthogonal components of the light beam amplitude;

- the optical anisotropic component is formed by spherolitic crystals $(l \sim 5 \ldots 10 \mu \mathrm{m} ; L \approx l)$ with predominantly circular birefringence $M_{44}(\theta)$, where $\theta$ is the polarization plane rotation angle.

\section{Optical realization of spatial-frequency of Fourier selection of endometrium}

Fig. 1 presents a diagram of laser Fourier polarimeter with spatial-frequency filtration [9]. Illumination of the sample under study was performed by the parallel $(\varnothing=$ $\left.10^{4} \mu \mathrm{m}\right)$ laser beam of $\mathrm{He}-\mathrm{Ne}(\lambda=0.6328 \mu \mathrm{m}, W=$ $5.0 \mathrm{~mW})$. The polarization light source consisted of quarter-wave plates 3,5 and polarizer 4 , it formed a right circularly polarized beam. Section of endometrium was placed in the focal plane of polarization microobjective 7 (focal distance $30 \mathrm{~mm}$, aperture 0.1 , magnification $4 \times$ ). Behind the Fourier focal plane the vignetting diaphragm was located, its size changed within the range of 2 to 300 pix. Polarization microobjective 8 (focal distance $30 \mathrm{~mm}$, aperture 0.1 , magnification $4 \times$ ) was located at the focal length from the frequency plane of lens 7 and, thus, performed inverse Fourier transform of the filtered out polarization field of laser radiation. The coordinate distribution of the intensity of these fields, polarizationally filtered by the quarter-wave plate 9 and polarizer 10, was registered in the plane of CCD-camera 11 (The Imaging Source DMK 41AU02.AS, monochrome 1/2" CCD, Sony ICX205AL (progressive scan); resolution $1280 \times 960$; light sensitive area size $7600 \times 6200 \mu \mathrm{m}$; sensitivity $0.05 \mathrm{~lx}$; dynamic range $8 \mathrm{bit}$; SNR 9 bit; deviation of photosensitive characteristics from linear is no more than $15 \%$ ). It provided the range of measuring the structural elements of the polycrystalline network with the resolution of 2 $2000 \mu \mathrm{m}[18-22]$.

The matrix element $M_{44}$ was calculated for each probing beam within every pixel $(m \times n)$

$M_{44}=\frac{I_{\otimes}^{\otimes}-I_{\oplus}^{\otimes}}{I_{\otimes}^{\otimes}+I_{\oplus}^{\otimes}}-0.5\left(\frac{I_{\circledast}^{0}-I_{\oplus}^{0}}{I_{\circledast}^{0}+I_{\oplus}^{0}}+\frac{I_{\otimes}^{90}-I_{\oplus}^{90}}{I_{\otimes}^{90}+I_{\oplus}^{90}}\right)$.

Here $I_{\otimes}^{\otimes ; 0 ; 90}$ and $I_{\oplus}^{\otimes ; 0 ; 90}$ are the intensities of spatial-frequency filtered images $(\otimes ; \oplus)$ for each polarization states of the probing beam $\left(\otimes ; \quad 0^{\circ} ; \quad 90^{\circ}\right)$.

To quantify the coordinate distributions, we used statistical analysis [7-10]. We calculated a set of statistical moments of the $1^{\text {st }}$ to $4^{\text {th }}$ orders $Z_{j=1 ; 2 ; 3 ; 4}$ by using the following algorithms:

$$
\begin{aligned}
& Z_{1}=\frac{1}{N} \sum_{i=1}^{N}\left|(q)_{i}\right|, Z_{2}=\sqrt{\frac{1}{N} \sum_{i=1}^{N}(q)_{i}^{2}}, \\
& Z_{3}=\frac{1}{\left(Z_{2}\right)^{3}} \frac{1}{N} \sum_{i=1}^{N}(q)_{i}^{3}, Z_{4}=\frac{1}{\left(Z_{2}\right)^{4}} \frac{1}{N} \sum_{i=1}^{N}(q)_{i}^{4} .
\end{aligned}
$$

The obtained data indicate the distribution histogram of random values of optically anisotropic structures in the plane of the histological section.

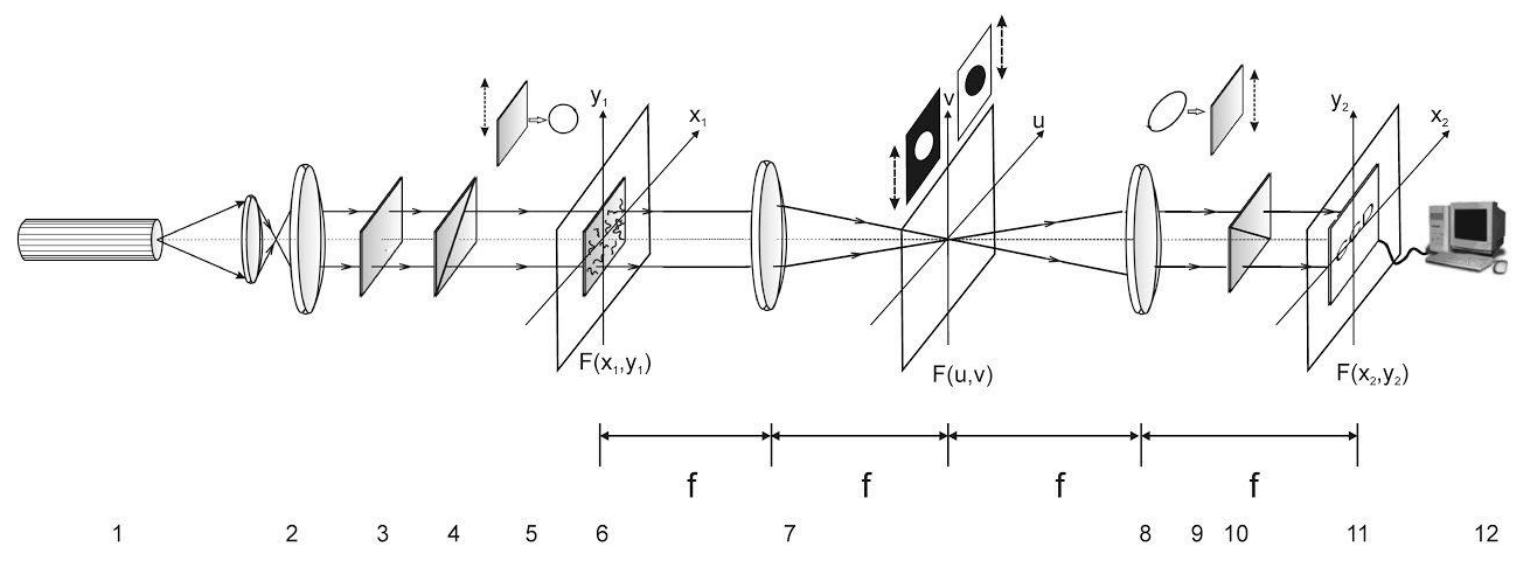

Fig. 1. Optical scheme of Fourier polarimeter, where 1 - He-Ne laser; 2 - collimator; 3 - stationary quarter-wave plate; 5, $9-$ mechanically movable quarter-wave plates; 4,10 - polarizer and analyzer, respectively; 6 - object of study; 7,8 - polarization microobjectives; 11 - CCD camera; 12 - personal computer. $F\left(x_{1}, y_{1}\right)$ - object plane, $F\left(x_{2}, y_{2}\right)$ - image plane, $F(u, v)-$ Fourier plane with the diaphragms. 


\section{Experimental results and discussion}

As objects of investigation, we chose two groups of optically-thin (extinction coefficient $\tau \approx 0.087 \ldots 0.098$ ) films with single scattering endometrium of donors (36 samples - group 1) and patients with pathology (36 samples - group 2).

The samples were prepared on a freezing microtome, using a standard technique. From the optical point of view, these samples are characterized by transformation of polarization without depolarization.

Fig. 2 shows the classic microscopic images of samples of both groups.

As can be seen, the coordinate large-scale structure of these classic microscopic images is similar to each other. This fact makes it difficult for histological differentiation of endometrium.

\section{Spatial-frequency Fourier polarimetry of linear birefringence in endometrium}

For the purpose of choosing optimal conditions of spatial-frequency filtration, the following range $\Delta r=$ $2 \ldots 50$ pix of possible sizes of the vignetting diaphragm.

The criterion for choosing the diaphragm size is the simultaneous change of the set of statistic moments $Z_{j=1 ; 2 ; 3 ; 4}$ of $M_{44}$. In our case the optimal size was $\Delta r=$ 30 pix.

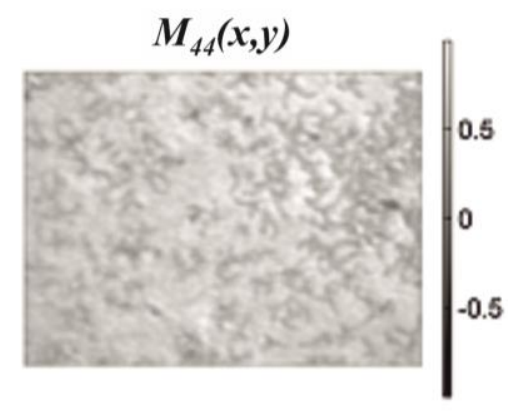

1)

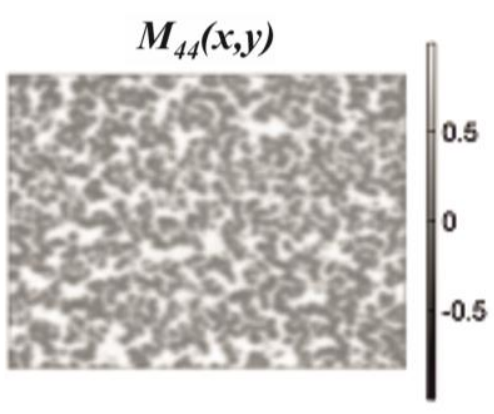

3)

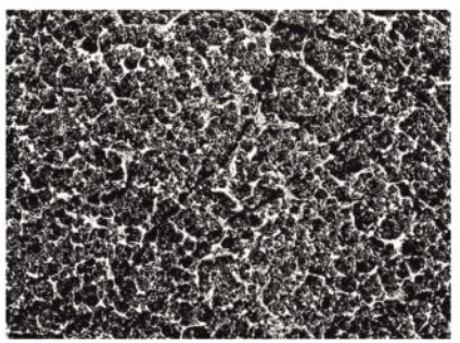

a)

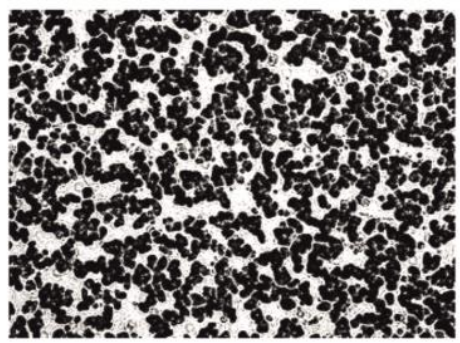

b)

Fig. 2. Microscopic images of the endometrium: group 1 (a) and group $2(b)$

This geometric size was chosen for comparative investigations of optical anisotropy of the fibrilar networks of the endometrium tissue that is characterized by coordinate distributions of rotational invariant $M_{44}(\delta)$ (Fig. 3).

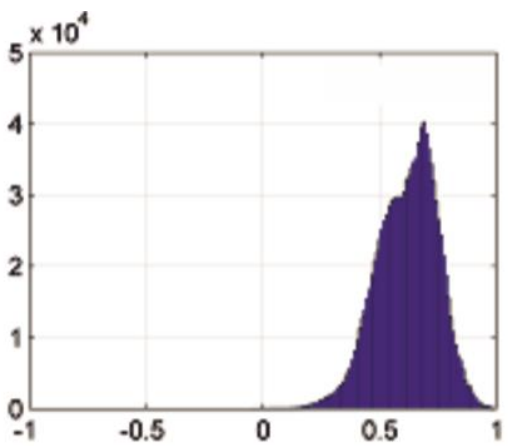

2)

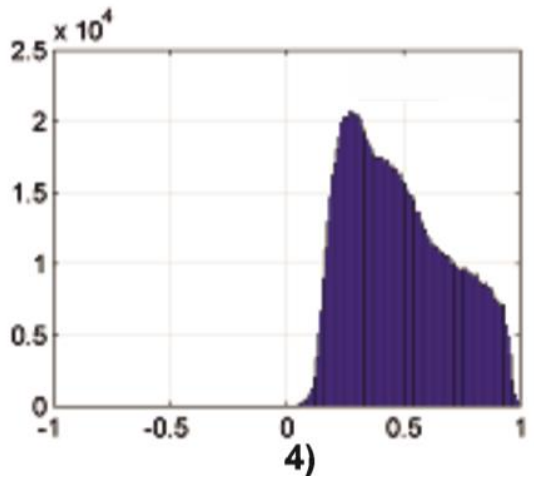

Fig. 3. "Low-frequency" coordinate maps $M_{44}(\delta)(1),(3)$, histograms (2),(4) of linear birefringence distribution in the endometrium of the group 1 (1), (2) and group 2 (3),(4). 
A comparative analysis of aggregate parameters that characterize matrix maps $M_{44}(\delta)$ of linear birefringence of large-scale optically anisotropic networks of the endometrium samples revealed some discrepancies between them. Namely, the main extrema of histograms in the distributions of random matrix maps $M_{44}(\delta)$ values for histological sections of both types are localized in different areas. Thus, the most probable $M_{44}(\delta) \sim 0.3$ value is for the group 1 (Fig. 3(2)); for the sample $2 \quad M_{44}(\delta) \sim 0.6 \quad$ (Fig. 3(4)). The revealed peculiarity, in our opinion, is related with a more developed structure of the endometrium of patient with pathology. The quantitative differences between matrix maps $M_{44}(\delta)$ of the protein matrices of the endometrium of both types illustrate average values and standard deviations of the set of statistic $Z_{i=1 ; 2 ; 3 ; 4}$ parameters summarized in Table 1. Also, let us introduce the specificity parameter $S p=a(a+b)^{-1} \quad(a, b$ are the numbers of the correct and incorrect diagnoses within each group) in differentiation of normal endometriosis.

A comparative analysis of the data of laserfrequency Fourier polarimetry for linear birefringence of the endometrium in both groups of patients revealed sensitivity to differentiation of these states of all the statistic moments of the $1^{\text {st }}$ to the $4^{\text {th }}$ orders with the specificity level $S p \sim 78 \ldots 83 \%$ (printed in grey color in Table 1).

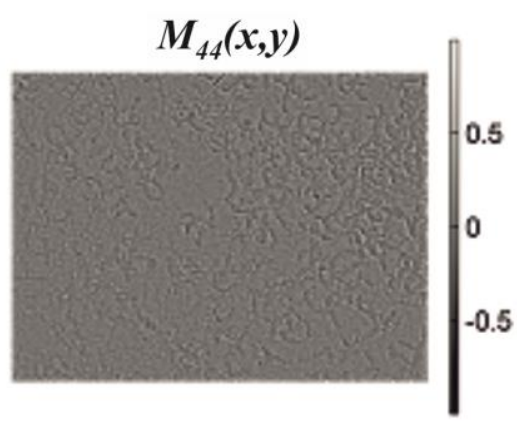

1)

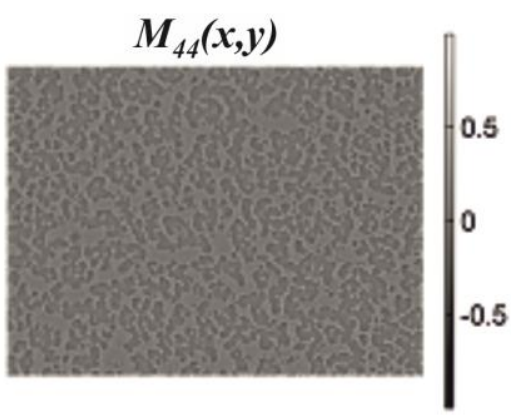

3)
Table 1. Parameters of the statistic structure of matrix maps $M_{44}(\delta)$ for linear birefringence of endometrium.

\begin{tabular}{|c|c|c|c|}
\hline \multirow{2}{*}{ Parameters } & \multicolumn{2}{|c|}{$M_{44}(\delta)$} & \multirow{2}{*}{$S p$} \\
\cline { 2 - 3 } & normal & pathology & \\
\hline$Z_{1}$ & $0.23 \pm 0.059$ & $0.52 \pm 0.11$ & $78 \%$ \\
\hline$Z_{2}$ & $0.12 \pm 0.022$ & $0.15 \pm 0.031$ & $63 \%$ \\
\hline$Z_{3}$ & $1.26 \pm 0.37$ & $0.78 \pm 0.14$ & $83 \%$ \\
\hline$Z_{4}$ & $1.59 \pm 0.28$ & $1.13 \pm 0.19$ & $79 \%$ \\
\hline
\end{tabular}

\section{Spatial-frequency Fourier polarimetry of circular birefringence in endometrium}

Diagnostic possibilities for differentiation of pathologically changed samples of endometrium tissue, using the method of high frequency spatial-frequency Fourier-domain polarimetry of circular birefringence employing opaque filter, illustrate probable dependences of matrix maps $M_{44}(\theta)$ and are adduced in the series in Fig. 4.

Comparing the findings about the structure of the distributions of circular birefringence index under the conditions of high frequency filtration of laser field radiation, it is the range of expansion of the random values that changes $M_{44}(\theta)$ for a histogram of the matrix map at the expense of a "pathologic" increase of the endometrium tissue.
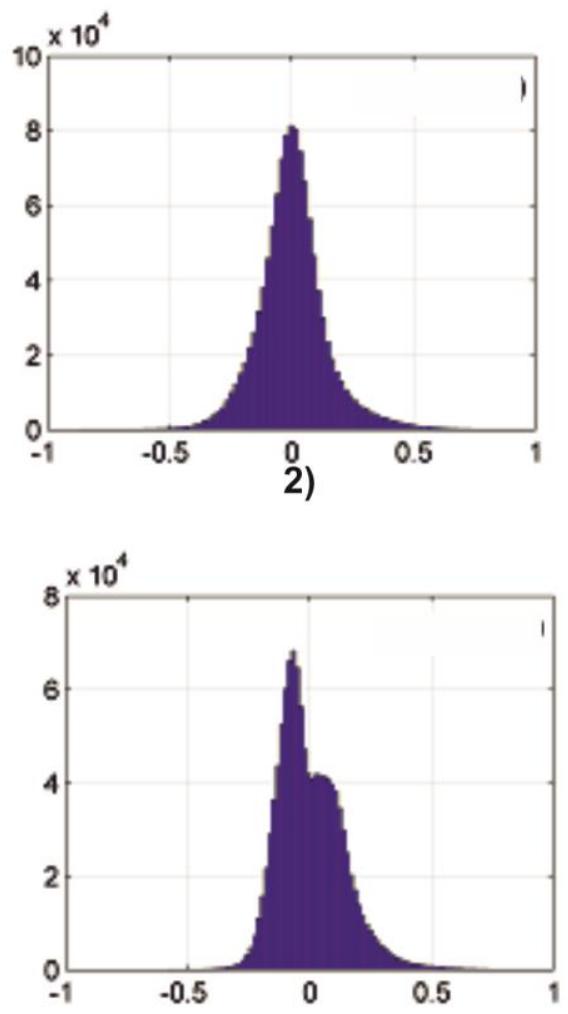

4)

Fig. 4. "High-frequency" coordinate maps $M_{44}(\theta)(1),(3)$, histograms (2),(4) of the linear birefringence distribution of the endometrium samples in the group $1(1),(2)$ and group $2(3),(4)$. 
Table 2. Parameters of the statistic structure of matrix maps $M_{44}(\theta)$ for circular birefringence of endometrium.

\begin{tabular}{|c|c|c|c|}
\hline \multirow{2}{*}{ Parameters } & \multicolumn{2}{|c|}{$M_{44}(\theta)$} & \multirow{2}{*}{$S p$} \\
\cline { 2 - 3 } & normal & pathology & \\
\hline$Z_{1}$ & $0.09 \pm 0.011$ & $0.11 \pm 0.017$ & $55 \%$ \\
\hline$Z_{2}$ & $0.15 \pm 0.018$ & $0.18 \pm 0.031$ & $61 \%$ \\
\hline$Z_{3}$ & $0.17 \pm 0.021$ & $0.48 \pm 0.39$ & $89 \%$ \\
\hline$Z_{4}$ & $0.38 \pm 0.043$ & $0.58 \pm 0.071$ & $78 \%$ \\
\hline
\end{tabular}

Quantitative differences between matrix maps $M_{44}(\theta)$ of circular birefringence of collagenous networks of both types illustrate average values and standard deviations of statistic set $Z_{i=1 ; 2 ; 3 ; 4}$ parameters adduced in Table 2.

A comparative analysis revealed the following condition with a high level of specificity $(78 \% \leq$ $S p \leq 83 \%$ ) of uterus tissue parameters (depicted in grey color in Table 2):

- $\quad$ statistic moments of the $1^{\text {st }}$ and $2^{\text {nd }}$ of orders $\theta(m \times n)$,

- the differences between the values are within the range from 2 to 3.5 times.

\section{Conclusions}

A set of criteria for polarization-phase differentiation of endometrium (normal and endometriosis) has been revealed and substantiated:

- $\quad$ statistical moments of the $1^{\text {st }}-4^{\text {th }}$ orders that characterize distribution of azimuthally stable matrix element $M_{44}(\delta)$ are defined by linear birefringence of the endometrium;

- $\quad$ statistical moments of the $1^{\text {st }}-4^{\text {th }}$ orders that characterize distribution of azimuthally stable matrix element $M_{44}(\theta)$ are defined by circular birefringence of the endometrium.

\section{Acknowledgement}

This work was supported by the grants № 0113 U003239 and № 0112U002336 from the Ukrainian Foundation for Basic Researches.

\section{References}

1. O.V. Angelsky, V.P. Pishak, A.G. Ushenko, Yu.A. Ushenko, Statistical and fractal structure of biological tissue Mueller matrix images, in: Optical Correlation Techniques and Applications, ed. by O. Angelsky. SPIE Press, Bellingham, 2007, p. 213-266.

2. O.V. Angelsky, A.G. Ushenko, Yu.A. Ushenko, V.P. Pishak, A.P. Peresunko, Statistical, Correlation and Topological Approaches in Diagnostics of the Structure and Physiological
State of Birefringent Biological Tissues. Handbook of Photonics for Biomedical Science, ed. by V.V. Tuchin. CRC Press, Taylor\&Francis group, Boca Raton, London, New York, 2010, p. 283-322.

3. Y.A. Ushenko, T.M. Boychuk, V.T. Bachynsky, O.P. Mincer, Diagnostics of Structure and Physiological State of Birefringent Biological Tissues: Statistical, Correlation and Topological Approaches. Handbook of Coherent-Domain Optical Methods. Springer Science+Business Media New York, 2013, p. 107-148.

4. J.M. Bueno, J. Jaronski, Spatially resolved polarization properties for in vitro corneas // Ophthal. Physiol. Opt. 21(5), p. 384-392 (2001).

5. M. Shribak, R. Oldenbourg, Techniques for fast and sensitive measurements of two-dimensional birefringence distributions // Appl. Opt. 42, p. 3009-3017 (2003).

6. M.H. Smith, Interpreting Mueller matrix images of tissues // Proc. SPIE, 4257, p. 82-89 (2001).

7. E.I. Olar, A.G. Ushenko, Y.A. Ushenko, Polarization correlation measurements of the phase tomograms of optically anisotropic biofractals // LASER PHYSICS-LAWRENCE, 14(8), p. 11151121 (2004).

8. O.V. Angelsky, G.V. Demianovsky, A.G. Ushenko, D.N. Burkovets, Y.A. Ushenko, Wavelet analysis of two-dimensional birefringence images of architectonics in biotissues for diagnosing pathological changes // J. Biomed. Opt. 9(4), p. 679-690 (2004).

9. O.V. Angel'skiň, A.G. Ushenko, S.B. Ermolenko, D.N. Burkovets, Yu.A. Ushenko, O.V. Pishak, Polarization-based visualization of multifractal structures for the diagnostics of pathological changes in biological tissues // Optika i Spektroskopiya, 89(5), p. $799-804$ (2000), in Russian.

10. R. Jóźwicki, K. Patorski, O.V. Angelsky, A.G. Ushenko, D.N. Burkovets, Automatic polarimetric system for early medical diagnosis by biotissue testing // Optica Applicata, 32(4), p. 603 612 (2005).

11. E.I. Olar, A.G. Ushenko, Y.A. Ushenko, Correlation microstructure of the Jones matrices for multifractal networks of biotissues // LASER PHYSICS-LAWRENCE, 14(7), p. 1012-1018 (2004).

12. S. Yermolenko, A. Ushenko, P. Ivashko, F. Goudail, I. Gruia, C. Gavrilă, Spectropolarimetry of cancer change of biotissues // SPIE Proc. 7388, Correlation Optics 2009, 73881D-73881D-7 (2009).

13. J.W. Goodman, Statistical properties of laser speckle patters. In: Laser Speckle and Related Phenomena. Ed. J.C. Dainty. Berlin, SpringerVerlag, p. 9-75, 1975.

14. O.V. Angelsky, P.V. Polyanskii, C.V. Felde, The emerging field of correlation optics // Optics and Photonics News, 23(4), p. 25-29 (2012). 
15. O.V. Angelsky, A.Ya. Bekshaev, P.P. Maksimyak, A.P. Maksimyak, S.G. Hanson, C.Yu. Zenkova, Self-diffraction of continuous laser radiation in a disperse medium with absorbing particles // Opt. Exp. 21(7), p. 8922-8938 (2013).

16. Yu.A. Ushenko, Yu.Ya. Tomka, A.V. Dubolazov, Laser diagnostics of anisotropy in birefringent networks of biological tissues in different physiological conditions // Quantum Electronics, 41(2), p. 170-175 (2011).

17. Yu.A. Ushenko, Yu.Ya. Tomka, A.V. Dubolazov, O.Yu. Telenha, Diagnostics of optical anisotropy changes in biological tissues using Müller matrix // Quantum Electronics, 41(3), p. 273-277 (2011).

18. O.V. Angelsky, Yu.A. Ushenko, A.V. Dubolazov, O.Yu. Telenha, The interconnection between the coordinate distribution of Mueller-matrixes images characteristic values of biological liquid crystals net and the pathological changes of human tissues // Adv. Opt. Technol. 2010, Article ID 130659, 10 pages (2010).
19. Yu.O. Ushenko, Yu.Ya. Tomka, O.V. Dubolazov, V.O. Balanets'ka, A.V. Karachevtsev, A.P. Angelsky, Wavelet-analysis for laser images of blood plasma // $A E C E$ - Adv. in Electr. and Comput. Eng. 11(2), (2011).

20. V.T. Bachinsky, Yu.O. Ushenko, Yu.Ya. Tomka, O.V. Dubolazov, V.O. Balanets'ka, A.V. Karachevtsev, Wavelet analysis for polarization maps of networks formed by liquid biological crystals in blood plasma: statistical and fractal approaches // Semiconductor Physics, Quantum Electronics \& Optoelectronics, 13(2), p. 189-201 (2010).

21. Yu.A. Ushenko, Yu.Ya. Tomka, A.V. Dubolazov, V.A. Balanetskaya, V.P. Unguryan, N.I. Zabolotna, B.P. Oleinichenko, Mueller-matrix diagnostics of optical properties inherent to polycrystalline networks of human blood plasma // Semiconductor Physics, Quantum Electronics \& Optoelectronics, 14(1), p. 98-105 (2011).

22. Yu.O. Ushenko, O.V. Dubolazov, A.O. Karachevtsev, M.P. Gorsky, Yu.F. Marchuk, Wavelet analysis of Fourier polarized images of the human bile // Appl. Opt. 51, p. C133-C139 (2012). 